\title{
The Emergence of AI and IoT on Cloud Computing: Evolution, Technology, Future Research and Challenges
}

\author{
Amedonu Debrah King Sena Bo Cuimei Kyei Baffour Boafoh \\ Department of Computer Science, Nanjing Technology University, 30 South Puzhu Road, Pukou, District \\ Nanjing, Jiangsu Province 211800, China
}

\begin{abstract}
Cloud computing is being widely promoted as the following paradigms and business solutions to the problems that most enterprise Information Technology (IT). Today it plays an important role in modern society and allows a wide range of applications from the infrastructure of social networks such system must cope with the increasing use of various loads and reflects the dependence of the interaction and community automated computer systems while the satisfactory quality of service $(\mathrm{QoS})$ guarantees. Cloud computing is beneficial to the owner of the company, as it eliminates the need for operators to plan ahead for the provision of resources, enabling organizations to start small and increase the resource only if there is an increased demand for services. To understand the current and future challenges of a system of this type, there is a need to identify the key technology for future applications. This work aims to study the history of computing and identify how the use of Artificial intelligence (AI) and Internet of things (IoT) influence cloud computing in order to solve complex problems in the next generation of computing. In addition, the influence of new paradigms and technologies on Cloud Computing were identified and discussed with future research opportunities and an open challenge stand out. Furthermore, this article also provides a conceptual model for cloud futurologist to explore the influence that paradigms and emerging technologies to the evolution of cloud computing are proposed.
\end{abstract}

Keywords: Cloud Computing; Paradigm; Information Technology; Internet of Things; Artificial Intelligence; Quality of Service.

DOI: $10.7176 / \mathrm{CEIS} / 10-7-03$

Publication date: November 30th 2019

\section{Introduction}

Cloud computing (CC) is the paradigm of IT services that enable users to get on-demand network access to a shared pool of resources is configured computing such as servers, storage, and applications [1] and also provide solutions that are flexible and cost-effective for many services through Internet [2]. Due to the rapid growth of cloud computing, has been adopted as an important utility in all aspects of society, from academia, government and industry. Characteristics such as dynamic cloud computing, metered access to a shared pool of computing resources have enabled the creation of new technologies and paradigms to meet the demands of emerging applications including scientific, health, agriculture, smart city and traffic management [3, 4]. The essential characteristics of the cloud-computing model include on-demand self-service, rapid elasticity, resource pooling and access to an extensive network. Cloud computing has gained a lot of popularity, which is mainly due to the following reasons, as discussed in [5]: advance commitments (i) of cloud computing have been eliminated by the end-user. (ii) Cloud computing has eliminated the overhead of manual planning, providing resources that are available on-demand, self-service, and the ability to scale as needed. Pay-as-you-go model has enabled the company to start small and increase its computing resources only when needed. Cloud computing services are set as, Software as a Service (SaaS), Platform as a Service (PaaS), Infrastructure as a Service (IaaS), Identity as a Service (IDaaS) and Networks as a Service (Naas). Cloud Storage as a service is a growing trend with features such as elastic, pay-as-you-go, business continuity with long-term retention and risk mitigation through disaster recovery [2].

Nowadays, to improve the organization's business strategies using historical data analysis techniques on them [6]. Some business sectors eg telecommunications and e-health have compliance requirements that bind them to keep historical data for a certain period [7]. Currently, the well-known cloud providers like Facebook, Google and Amazon Cloud utilize large-scale Data Center (CDC) for the provision of heterogeneous Quality of Service (QoS) requirements $[8,9]$. In addition, the cloud computing platform is able to provide a unified interface over heterogeneous resources found on the Internet of Things (IoT) based application that improves the reliability of cloud services [10]. There is a need to sign a Service Level Agreement (SLA) between cloud users and providers to deliver the necessary services within the stipulated time and budget based QoS parameters such as time limits, reliability, availability, cost, safety and privacy [11]. To improve the reliability of cloud computing systems, there is a need to develop a new fault-tolerant mechanism, which can maintain the quality of cloud services for the hardware or software errors [12]. Furthermore, the new programming model such as serverless computing [135] allows new patterns of resource consumption, autonomously driven by the utilization of applications [6]. Lightweight virtualization technology provided by the container, it can improve utilization in the cloud, and enable 
lower latency providing application environment [13]. Furthermore, the advent of fog computing reduce latency and response time in the processing of IoT devices but is still researching the challenges in this domain are not resolved effectively. The new provision of resources and scheduling of the policies needed to fog and cloud computing using Artificial Intelligence (AI) based on in-depth learning techniques to predict in advance the resource requirements for the different geographical sources [14, 15]. Cloud computing is emerging as a new tool for solving complex challenges faced by researchers Earth Sciences both in the context of computing and analysis [16]. However, innovations in the field of cloud computing require a revisit of paradigms (Artificial intelligence and Internet of Things) on cloud computing. Therefore, there is a requirement for a systematic review to evaluate, upgrade, and integrate the existing research presented in this field with respect to the emerging paradigms and technologies such as IoT and AI. This systematic review presents an updated study to evaluate and discover the research challenges based on the available existing research along with the evolution and history of computing systems as per new frontiers as an amalgamation of these technologies having a high impact on cloud computing and related domains.

The objective behind this systematic review is to study the history of computing and identify how the rise of $\mathrm{AI}$ and IoT influence cloud computing in order to solve complex problems in the next generation of computing. Also, a conceptual model to explore the influence of paradigms and emerging Artificial Intelligence and IoT on the evolution of cloud computing is proposed.

This study is structured as follows. Section 2 presents Computing paradigm: Definitions, evolution and technologies. Section 3 describes the controller (Artificial Intelligence and IoT) cloud computing. Section 4 presents the impact of the new paradigm of technology and cloud computing with future research opportunities and an open challenge. Section 5 presents a conceptual model for cloud futurology. Finally, section 6 research conclusions are drawn.

\section{Computing paradigms: definition, Evolution and Related Technologies}

It made possible the technological progress in network speed and bandwidth of the emergence and evolution of distributed computing. It began in the 1960s in the message-passing communication between two or more computing resources. Then the local area network, ARPANET, and especially the introduction of email service marked the 1970s The use of distributed computing has undergone a major expansion that benefits from the availability of computing resources powerful and network speed to the public at low cost. Solving large complex problems is considered extremely difficult to resolve became possible. In this section, the main distributed computing paradigms shown in Figure 1.

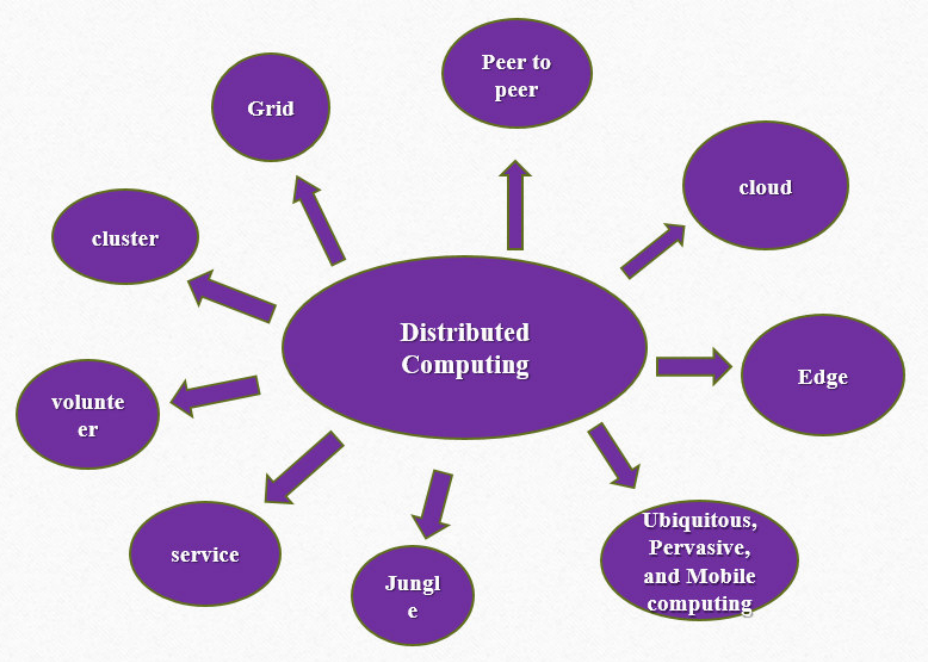

Figure 1. Main distributed computing paradigms

\section{Cluster Computing:}

Clusters are groups of independent computers collocated interconnected via a high-speed network [17], working closely so that in many ways, they form a single computer [18]. Clusters are naturally suited to systems that perform a large number of independent (or nearly independent) small stones [19]. Cluster components are generally, but not always, linked by fast through LAN [20]. All connected or loosely computers are considered a single system. The success of cluster computing is based on the use of low cost and commercial equipment and widely used software. And its main objective in the high-performance computing cluster is to reduce the execution time $[3,21]$. In practice, each node in a cluster runs a local copy of a single processor operating system. Therefore, any management at the system must be done by "middleware" above the operating system [22]. 


\section{Grid Computing}

Grid computing is the sharing of computing resources (computers, clusters, parallel machines, ...) by a number of people and institutions in a flexible and secure environment [20]. It creates the illusion of a simple selfmanagement and powerful virtual machine on a large collection of heterogeneous systems and resources [23]. The objective is to enable the sharing of resources and coordinated problem-solving in dynamic, multi-institutional virtual organizations [24]. Clabby Analytics defines the grid as a distributed network architecture that detects and operates unused computing/storage resources found in a distributed computing environment [25]. Grid computing can be used in a variety of ways to approach various types of application requirements. There are a large number of projects in the world of work on the development Grids for different purposes at different scales to provide nontrivial services to users [26].

\section{Peer to Peer}

In the peer-to-peer computing in contrast to client-server systems, each node acts as both a client and a server providing a part of system resources [27]. All client machines operate independently to join or leave the system freely. This implies that no master-slave relationship between the peers. No central coordination and no central database is needed. In other words, no peer machine has a global vision of the entire P2P system. The system is self-organization with distributed control without the need for central coordination by the central server [24]. The resources and shared services include the exchange of information, processing cycles, the cache storage and disk storage for files [28]. They are used in many application areas such as data transfer and storage of data, but the concept was popularized by file-sharing systems such as music-sharing application Napster [29].

\section{The Cloud Computing}

A fundamental characteristic of cloud computing is the capability to deliver, on-demand, a variety of IT services that are quite diverse from each other. This variety creates different perceptions of what cloud computing is among users. Despite this lack of uniformity, it is possible to classify cloud computing services offerings into three major categories:

- Software as a service (SaaS): The consumers are only provided with the capability to run the applications of the provider, but they have no control over the cloud infrastructures like operating system, servers, or storage. It also offers software functionality as a service without any maintenance and initial cost with high quality and an example of SaaS is Gmail [30].

- Platform as a service (PaaS): The consumers have the capability to deploy either own or acquired applications to the cloud. The consumer does not have any control over the cloud infrastructure but has control over the deployed application and an example of PaaS is Microsoft [31].

- Infrastructure as a service (IaaS): The consumers can use the applications provided on the cloud without the need to download the application to the consumer's computer. Consumers can manage the underlying infrastructure at the cloud such as virtual machines, the operating systems, and other resources an example of IaaS is Amazon[32].

\section{Edge Computing}

Edge computer is a natural extension of the architecture Content Delivery Network (CDN) [17] [33]. Davis [33] again pushes the application logic and processing the underlying data enterprise data centers to proxy servers to the "edge" of the network to achieve scalable web services and highly available [34] and for better efficiency and performance [34]. Computer Edge has several applications in different fields [35]. For G. Roussos et al [36] this displacement toward the edge of the computer is typical in widespread applications of calculation and is observed in a variety of related situations, especially with the wireless sensor networks.

\section{Ubiquitous, Pervasive, and Mobile computing}

Ubiquitous computing, which means "existing everywhere," is the method of enhancing computer use by making many computers available in the physical environment, but making them effectively invisible to the user $[37,38]$. Its beginning was in laboratory and imaging electronics research Centre Xerox Palo Alto in the early 80s and it provides a framework for new and exciting research across the spectrum of computer science [37, 39, 40]. Ubiquitous began Palo Alto Research Center of Xerox. In 1991, Mark Weiser, director of technology, described the ubiquity of personal computers: "The most profound technologies are those that disappear They are woven into the fabric of everyday life until they are impossible to distinguish thereof [41]. His extensive use of video and audio, including voice communication, transform electronic interfaces in interpersonal relationships [38].

\section{Jungle computing}

Jungle computing was introduced in 2011 by J. F. Seinstra et al [42] refers to the use of diverse, distributed and highly non-uniform high-performance computing systems to achieve peak performance [24]. This resulting distributed system, called Computing jungle system is both heterogeneous and hierarchical, potentially including grids, clouds, autonomous machines, clusters, office screens, mobile devices and supercomputers, possibly with accelerators such as GPU [43].

\section{Volunteer Computing}

Volunteer computing is often called desktop computing because it uses desktop PCs as computing resources 
underlying [44]. Most volunteer computing platforms have the same structure: a client program runs on the volunteer computer. It regularly contacts the project servers on the Internet, demanding jobs and return the results of completed jobs.

\section{Service computing}

Service computing (or service-oriented computing) is "a way to develop services application systems as the basic elements" [45]. It is an effective approach to IT paradigm distributed and one of the key cloud computing used to model, build, operate and manage business services [46]. The service model calculation is based on a service registry, service consumer and service provider. It leads to a distributed computing model of interactions based on dynamic service. First, the service provider registers itself in the registry. The consumer services then discovered the register of the service and use it.

\section{The Internet of Things and Artificial Intelligence of cloud computing and their related works}

Cloud computing becomes intelligent computing with the emergence of innovative technologies and paradigms such as Artificial Intelligence and Internet of Things.

\subsection{Artificial Intelligence}

$\mathrm{AI}$ is the process to generate intelligent machines and perform tasks of human intelligence, such as decision making, speech recognition, and so on. This process of learning, reasoning and self-correction. Learning is the acquisition of information and data usage rules; the reasoning is the rules to reach approximate or final conclusions. The integration of AI provides the path to the collection of information up, analyze and appropriate learning that is used for many applications such as health, smart home, smart agriculture, and the intelligent vehicle and so on. It takes raw data as input and performs decision making, collecting raw data or input and finally gives the maximum power or the chances of success of an intention or a particular purpose [47]. There was more work to draw artificial intelligence techniques party to improve the performance of fog and cloud systems [48-51]. Various studies focus on best planning policies for cloud, virtualization algorithms, and distribution systems, among others. They use research methods such as genetic algorithms, machine learning and even supervised a deep reinforcement learning to maximize their objective functions $[52,53]$. Amnesty International provides a lucrative avenue for optimizing large systems with huge amounts of data with the simplicity of engineering and efficiency by enabling automated decision making instead of human coded heuristics that provide decisions that are more effective quickly. Cloud computing is growing rapidly and become an important part of CDCs prominent industries such as Facebook, Microsoft, Google, Amazon [54].

Gil et al. [55] studied regarding intelligent machines it eliminates in many areas of work of a human being such as medical science, automatic detection devices, automatic driving vehicles and kitchen.

\subsection{Internet of Things (IoT)}

Information of things is a paradigm for the connection between the communication devices and human Internet as Internet Everything (IoE). Peripherals are capable of communicating with each other; it comprises smart home, smart vehicle and automotive [56]. As such, the IoT applications are able to operate across heterogeneous domains and enable rich analysis and management of complex interactions [13]. Thus, the IoT devices and the service are able to meet the challenges in a wide range of application areas, including eHealth, infrastructure, building management systems, manufacturing and transport [57, 58]. The Internet of things has several features of central devices for their operation. This includes (i) systems are often very dynamic and network members face volatility, where a device may appear and reaper across multiple networks [59]. (ii) They are very heterogeneous in terms of computing power and functional capacity, and as such system has to cope with a limited processing, memory and persistent storage [60] and (iii) The systems are managed and controlled by multiple stakeholders, which requires federal mechanisms for the secure management of data collected IoT [58]. Historically IoT applications have offloaded processing, and persistent storage to cloud services, but the number of "things" grows, these services fail to support demand in real-time IoT devices [59, 61]. Intelligent e-health applications are able to monitor the data of the patient in real-time, collecting data from implantable devices and easy to wear forming personal networks [62]. Furthermore, Fog activated IoT systems are adaptable and can change their behaviour according to the state determined by the collected sensor data. For example, an intelligent gateway collecting samples from a pacemaker can increase its sample prior to a heart attack, detected by means of pre-processing the fog layer [63]. The Internet of energy paradigm (IoE) introduced the concept of smart grids and energy management [64]. In addition, such a grid can provide safer, more reliable and robust power delivery, to meet the changing demands of consumers [65]. Chung et al. [66] described that the Internet is a huge source of generation amounts of data from IoT applications quickly accumulated. Atzori et al. [67] identified three categories of IoT that are oriented Internetoriented sensors and knowledge. 


\section{The influence of New Paradigms and Technologies on Cloud Computing along with their future research opportunities and open challenges.}

Cloud computing is changing very rapidly and a various number of researchers and academicians working actively to solve existing research challenges in the field of cloud computing.

We also identified several areas of research related to cloud computing, which uses its available technologies and paradigms effectively to solve current problems. Figure 2 shows the research areas of the emergence of this study.

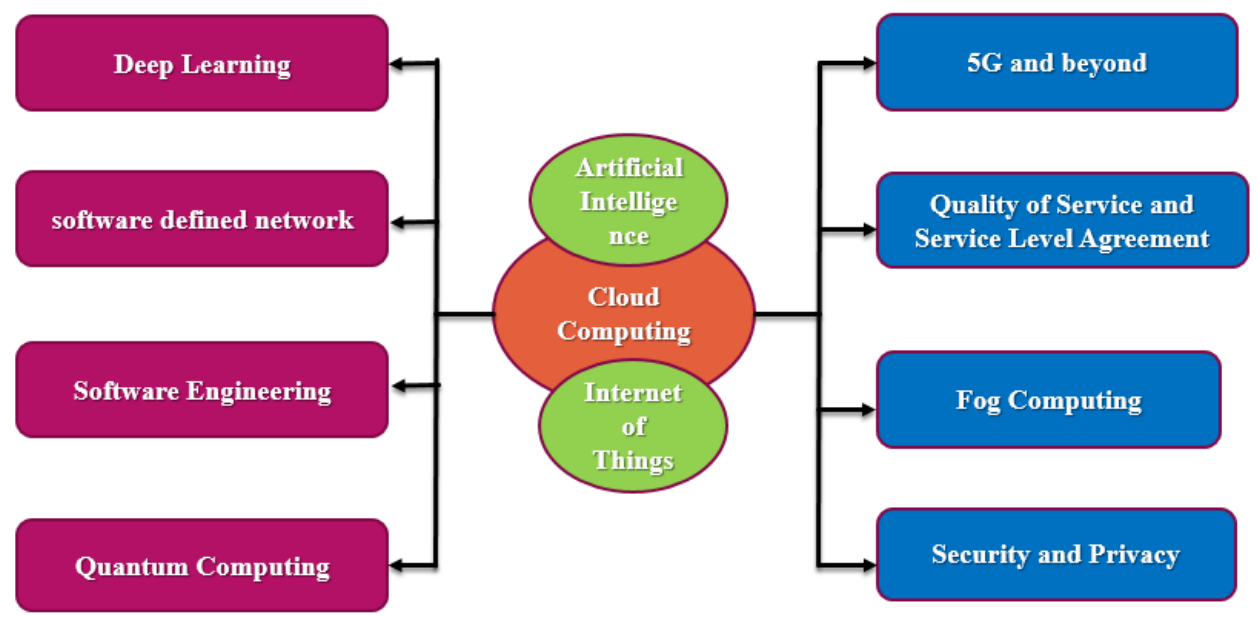

Figure 2: Study Areas of Emergence

We gave the foundation of paradigms and emerging technologies for researchers, academics and practitioners in this section and related future research directions and open challenges are presented at the end of each paragraph.

\subsection{Deep Learning}

Deep learning is a learning software engineering class machine that is based on learning from large data sets [52]. The heart of deep learning is to get interactive features high level from the raw data. Lately, deep learning was turned Reinforcement learning to help realize the field of deep learning reinforcement that offers hope in the development of the best models in the future [68]. The intersection of deep learning and cloud computing creates interesting applications in which the two fields complement each other should first discuss how cloud computing supports scientific data and move later to how deep learning was taking advantage of the various solutions to traditional problems in cloud computing [69, 70]. Moreover, Teerapittayanon. al [71] have demonstrated the use of edge devices and IoT end in the embodiment of a distributed formation of a depth of the neural network. Cloud computing also helps reduce power consumption deep neural networks, by reducing the size of the function obtained by fractionation of the network architecture between the mobile and cloud [72]. Nguyen et al. al [49] have developed a deep learning model to counter cyber-attacks in the context of mobile cloud kingdom. Li et al. al [73] have developed a system that uses the cloud and in-depth learning in order to minimize energy consumption by clouds clusters. Safety has always been the reason for adopting cloud impeding by users that can be treated using deep learning as many authors recently showed a persistent problem with cloud computing is the management and monitoring of large groups of clouds.

Future research directions and challenges for Deep Learning are summarized as follows:

1. How to enable deep learning on IoT devices to improve the actual performance of the world in artificial intelligence-based intelligent systems?

2. A system overall healthcare chip based on deep learning is necessary for automatic diagnosis of heart disease in IoT integrated fog and IT environments.

\section{2 software-defined network}

It is necessary to allow the virtualization network concept in the clouds is called software-defined network (SDN) and use NRS to cloud computing by extending the idea of virtualization of all cloud resources such as network the storage and calculation [74]. In addition, it improves the abstraction of physical resources and the automation and optimization of the configuration process. NRS paradigm offers a platform to allow flexibility or agility in the network, which can create a profitable communication between modern cloud data centers. In addition, computer NRS based cloud reduces energy consumption while optimizing network virtualization. Virtualization Network Features (VNF) is another emerging paradigm network that transmits network functions such as load balancing and intrusion detection Domain Name Service (DNS) when running software applications [75]. Moreover, VNF 
improves the elasticity of the network function and increases the flexibility and agility of the service, which further reduces the cost $[75,76]$. There are various research challenges are still open for academics and researchers. First, it is necessary to provide the security mechanism for cloud computing based on NRS to secure the transfer of data between different cloud data centers [77]. Adel al. [78] developed a low-cost micro-based Raspberry Pi-data Centre for cloud computing software-defined, reducing costs, but service reliability is still questionable. Second, the balance between the cost and the power consumption still exists because of the replication of NRS enabled cloud infrastructure. In the future, it is necessary to deploy a cloud-computing environment based on NRS, which can reduce energy consumption and increase reliability while providing a cost-effective way to network virtualization service.

Future research directions and challenges for the software-defined network are summarized as follows.

1. It is necessary to deploy a cloud-based environment using models of NRS learning AI, which can reduce energy consumption and increase reliability while providing network virtualization service profitably.

2. It is necessary to provide the security mechanism for cloud computing based on NRS for secure data transfer between devices using CDCs IoT.

\subsection{Software Engineering}

Software Engineering (SE) and cloud computing are very similar paradigms. For example- SE Service-Oriented combines the best features of the services, cloud computing, and gives several advantages to the software development process and applications. The only difference between services-oriented SE and cloud computing is that -the If service-oriented focuses on architectural design (service discovery and composition) and cloud computing focus on effective service delivery users through virtualization of flexible and scalable resource and load balancing [163]. Software Engineering is evolving not only hardware technologies but also involves customers and software developers [79]. Cloud computing and virtualization allow users to create virtual machines and cloud services for projects and software with automatic management [80]. With cloud services, software development teams can combine the development, testing and delivery processes seamlessly. Cloud computing can improve the software engineering process as follows:

- The development process can be accelerated. Cloud computing and virtualization offer sufficient computing resources, so developers can use multiple virtual machines rather than stick to a single physical machine.

- Cloud computing enables the development activity in a parallel manner, as a time to install the necessary applications can be reduced by getting the cloud services, which can lead to a more efficient development process.

Cloud computing offers new opportunities for software engineering researchers to explore the development of multilateral programs. Emerging technologies based on IoT and machine learning and artificial intelligence open a new software engineering research field and the major problem in these technologies manage the enormous amount and variety of data. This research also gives the opportunity for new research and new ways to manage data in the cloud, which translates to the start of improved technologies as fog Computer- first used by Cisco to expand infrastructure current cloud computing [81]. These new areas are new but highly dependent on software engineering.

Future research directions and challenges for software engineering are summarized as follows:

1. How to program the system "automatically" using artificial intelligence instead of writing software code manually?

2. 1. To study the feasibility of software engineering solutions based on IoT on how organizations can deliver high-value business through technological commitments and operating strategy at the same time can generate a return on investment (ROI) by effectively using the possibilities of IoT in business.

\subsection{Quantum Computing}

Quantum computing is the branch of computer science in which we operate and exploit the laws of quantum mechanics to process the data. Classical computers cannot solve the problems of a certain size and complexity. Quantum computers take advantage of the superposition of phenomena and entanglement to solve difficult problems [82]. Layering is a phenomenon in which the quantum computer can be in several states simultaneously. Although the entanglement is the phenomenon to be a strong correlation between two or more quantum particles which are inseparable, even if they are separated by a large distance. Because of these two principles, a quantum computer can make great numerical calculations simultaneously. [38] Think of it this way: while a conventional computer works with ones and zeroes, a quantum computer will have the advantage of using zeros and "overlays" of ones and zeros [83]. Some difficult tasks that have long been thought impossible (or "intractable") for conventional computers will be achieved quickly and efficiently by a quantum computer.

Future [82] addresses this area of research are:

1. Learning and AI: Quantum computing can transform camp of machine learning too. Object detection is 
very easy for human beings to collect different objects of photography. Programmers do not know how to write code that can infer many objects on their own. Machine learning is one approach to solving this problem algorithm that recognizes the objects to obtain a formation of large datasets. [84]. Quantum computers make these types of problems because they can easily make huge calculations simultaneously.

2. Simulation of Material: Quantum computers are simulating possible, materials, equipment simulation field can lead to development in various IoT based applications in the robotics industry, chemical and optical [85].

Future research directions and challenges for Quantum Computing are summarized as follows:

1. Quantum computing can transform domain field of machine learning too. Object detection in which it is very easy for a man to choose different objects of photography. As programmers, do not know how to write code that can deduct many items by itself. Learning the machine is one approach to solve the problem in which the algorithms recognize objects get training on large data sets.

2. Quantum computers are simulating possible, materials, equipment simulation field can lead to development in various IoT based applications in the robotics industry, chemical and optical.

\section{5 $5 \mathrm{G}$ and beyond}

NGN not only promise very high data rates and low latency, but also universal coverage and massive IoT. Today, the dense network deployment is one of the effective strategies to meet the requirements of capacity and the fifth generation of connectivity (5G) cellular system [86]. 5G is the fifth-generation cellular mobile communications, preceded by 4G systems (LTE / WiMax), 3G (UMTS) and 2G (GSM). 5G is expected to provide high-speed data rates, reduced latency, reduced power consumption, cost, the capacity of the system capacity, and the simultaneous massive connectivity device [87]. One of the main objectives of $5 \mathrm{G}$ networks to support applications that require a high-density device. In this respect, mass communications concepts typewriter (MMTC), Enhanced Mobile Broadband (EMBB) and ultra-reliable communications with low latency (URLLC) are being developed to support these applications $[87,88] .5 \mathrm{G}$ bring major advances in the network and communication systems by providing an ultra-high-speed data transmission which can be 100 times faster than existing 4G [89]. Edge computing as an evolution of cloud computing moves applications hosting paradigm of centralized data centers to the network edge, closer to the consumer and the data generated by applications [90]. Edge computing is considered one of the main factors to meet the demanding key performance indicators (KPIs) such as $5 \mathrm{G}$ up improved mobile broadband, low latency and massive connectivity. The revival of computationally intensive applications in the Internet of Things (IoT) era and the growing number of critical tasks in emerging networks is a major bottleneck in the design of communication systems in real-time, [88]. The advent of 5G and cloud computing will improve the capacity, functionality and flexibility of the network operators to offer a new range of services [86]. Data analysis on massive amounts of data collected from the massive number of sensors in the case Industrial use IoT can be managed profitably by analyzing data based $5 \mathrm{G}$ and $\mathrm{AI}$, because of its high-speed data and low latency promises. Future research directions and challenges for $5 G$ and beyond are as follows:

1. Data analysis on massive amounts of data collected from the massive number of sensors in the IoT cases of industrial use can be managed cost-effectively using artificial systems based 5G.

2. The applications of computing stimulating intensive in the IoT era and the growing number of tasks mission-critical in emerging $5 \mathrm{G}$ networks is a major bottleneck in the design of communication systems in real-time.

\subsection{Quality of Service and Service Level Agreement}

Quality of Service (QoS) is an important challenge for cloud computing systems that can predict system performance when running [9]. The QoS parameters such as execution time, cost, scalability, elasticity, latency, reliability, etc., can measure the performance of the computer system [91]. QoS parameters are defined using the Service Level Agreement (SLA), which is an official document signed between the user and the cloud provider based on various QoS parameters [157]. At that time, there are many IoT applications with different QoS parameters according to their field, purpose and the requirement with more stringent security requirements that can use blockchain and related technologies [92]. In addition, ALS can be measured using a measurement called SLA violation rate, which can estimate the actual SLA away from the necessary (Estimation / predicted) and decides the compensation for SLA violation [93]. QoS is gradually important when including cloud services, as the quality of service in damaging one of the services can dangerously affect the system's quality of service in its computer together. To provide an efficient cloud service, it is necessary to provide the required amount of cloud resources that can meet the quality of service of an application such as the budget, the response time and the time [94]. Therefore, cloud providers must ensure with enough supplies resources to avoid or reduce SLA violation rate to perform the user workloads into their deadline and budget. The future of the new generation of computer systems depends on the QoS resource management mechanisms, which can identify and meet the QoS requirements of the computer system $[9,54]$. There are various research challenges [62, 95-97] are in effect that prevents the realization 
of the quality of service effectively. First, there is an unavailability of cloud resources to run an application running, increasing the run time and reducing system performance. Second, it is necessary for an effective management mechanism SLA-aware resource reduces the SLA violation rate and maintain the performance of the computer system. Thirdly, there are different standards for different SLA cloud providers and there is a need for standard centralized SLA to achieve the common objective of the multi-cloud environment. Finally, it is necessary to find a compromise between the different QoS requirements due to a wide range of IoT applications running on cloud computing systems using AI-based techniques or prediction models supervision / unsupervised learning.

Future research directions and challenges for QoS and SLA are summarized as follows:

1. AI approaches have completely changed the landscape of the IoT applications, also mobile devices for transmission of application media of IoT has become very necessary for end-users.

2. It is necessary to find a compromise between the different QoS requirements due to a wide range of IoT applications running on cloud computing systems.

\subsection{Fog Computing}

Fog calculation is in the form of distributed computing paradigm that acts as an intermediate layer between the IoT devices and cloud data centers [13]. Fog is a cloud supplement to, not a replacement. Fog can never completely replace the cloud that we still need to manage large or complex data problem. The fog only provides more or less that cloud services are not provided within the same period or latency requirements [98]. Fog reduces latency for applications that require fast response time (as real-time applications, namely the circulation system, emergency system, health system, etc.) are going to fog services instead of cloud computing services. However, it fails when the data is very cumbersome to handle by its local nodes [91]. Producing huge data by IoT devices is also the reason to accept IT paradigm fog that these data cannot be processed by the current cloud system, even with high computing capacity [99]. Beyond low latency, various services such as support for mobility, security, good performance and low bandwidth requirement are also provided by the fog. Fog usually provides an intermediate layer rich in resources between the terminals and the cloud system to achieve the above goals. It acts as a bridge between end-users and the cloud system. Fog nodes are also attached to the cloud using the Internet to use its food services and computer storage and it generally analyzes the data produced by these end devices and sensors [100]. Most existing job scheduling algorithms are based on heuristics [101, 102]. Other works use adaptive techniques to optimize investment decisions and migration [48]. In this regard, AI-based techniques can be utilized to provide more efficient and robust algorithms to improve investment and tasks directed to user needs.

Future research directions and challenges for Fog Computing are summarized as follows:

1. State of the art AI techniques can be used for the appropriate task scheduling on heterogeneous fog environments.

2. Generic interfaces are required for the fog gateways to be able to interact with the plethora of IoT devices.

\subsection{Security and Privacy}

Recently, research and industry, there has been a massive transfer of personal computing in IoT, Edge and Cloud Computing to provide smarter and more effective services to end-users. For this great paradigm shift, many problems and challenges have emerged in the privacy and security for the data on these devices. Due to various characteristics of advanced IT as low latency, geographic distribution, mobility of the end device and the high treatment, heterogeneity, etc. [54,99]. The security and confidentiality properties must be more robust and versatile. In addition, the diversity of applications and the heterogeneity of devices, it is difficult to develop software platforms seamlessly connected. To study these security measures and concerns in cloud and the IT paradigm fog, the following factors become important: (1) The trust and confidence of end-users (2) source authentication Internode and validation (3) Impenetrable communications between sensors, computing and brokers nodes (4) identification and protection systems against malicious attacks [103]. Security mechanisms must also take into account the existence of mobile devices using these data centers.

Future research directions and challenges for Security and privacy are summarized as follows:

1. AI on the basis of the current security techniques can be explored as the safety analysis grouping based on a model that is useful in mitigating DDoS attacks in server systems and detection systems intrusion.

2. Due to the intrinsically decentralized nature of the fog of computers, many unforeseen security threats in the fog layer and IoT devices that are not a concern in cloud computing.

\section{A Conceptual Model for Cloud Futurology}

To solve the above problems, there is a conceptual model of the need to explore the influence of three new paradigms Internet of Things and artificial intelligence on the evolution of cloud computing. Figure 3 shows the conceptual model describing the effects of processing technologies and paradigms on the development of the cloud. The model incorporates the Internet of things and artificial to provide an overall view of an abstract design encompasses several areas of computer intelligence. Various components of the Internet of Things including 
sensors and actuators communicate with the gateway, all connected by the $5 \mathrm{G}$ technology. The user interacts with the model using the application interface in the gateway device. Layer gateway also contains the engine basic data preprocessing and various IoT applications for generating jobs and collecting results transparently. Jobs are sent and managed nodes agent authenticate payments and other transactions with power modules and sophisticated resource management, backed fault tolerance and security systems. These nodes are also connected to the controllers and external database through NRS. Software engineering allows the end to end integration with cloud resource availability deployments based server without server frameworks. The model is enhanced by the technology of quantum computing. In general, the model incorporates and allows calculation using a lot of technological advances and provides an enhanced and holistic setting for the new generation of IT environments.

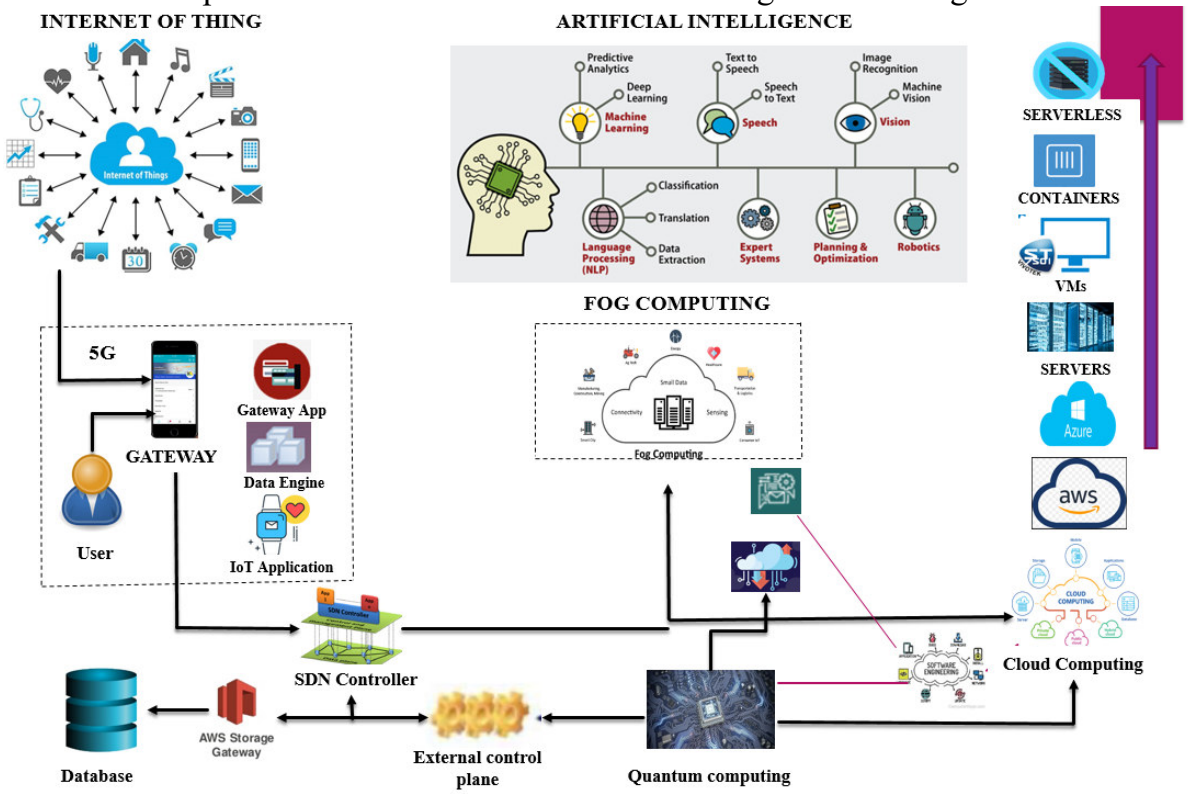

Figure 3: A Conceptual model for Cloud Futurology.

\section{Conclusions}

Cloud computing is an emerging paradigm, enabling on-demand, metered access to computing resources driving technological innovation and enabling geographically distributed applications.

In this review study, a systematic reviewed of computing paradigms and technologies and the influence of IoT and Artificial Intelligence to the evolution of cloud computing have been presented. Further, the research areas related to the influence of new paradigms and technologies on cloud computing have been identified, discussed and their future research opportunities and open challenges are highlighted. Also, a conceptual model to explore the influence of emerging paradigms (IoT and Artificial Intelligence) on the evolution of cloud computing was proposed. We hope that this systemic review will be beneficial for other authors who want to do study in any area concerning cloud computing.

\section{Reference}

[1] "Appendix C - The NIST Definition of Cloud Computing," in Application Performance Management $(A P M)$ in the Digital Enterprise, R. Sturm, C. Pollard, and J. Craig, Eds., ed Boston: Morgan Kaufmann, 2017, pp. 267-269.

[2] S. Nepal, S. Chen, J. Yao, and D. Thilakanathan, "DIaaS: Data integrity as a service in the cloud," in 2011 IEEE 4th International Conference on Cloud Computing, 2011, pp. 308-315.

[3] B. P. Rimal, E. Choi, and I. Lumb, "A taxonomy and survey of cloud computing systems," in 2009 Fifth International Joint Conference on INC, IMS and IDC, 2009, pp. 44-51.

[4] T. L. Casavant and J. G. Kuhl, "A taxonomy of scheduling in general-purpose distributed computing systems," IEEE Transactions on software engineering, vol. 14, pp. 141-154, 1988.

[5] A. Fox, R. Griffith, A. Joseph, R. Katz, A. Konwinski, G. Lee, et al., "Above the clouds: A berkeley view of cloud computing," Dept. Electrical Eng. and Comput. Sciences, University of California, Berkeley, Rep. UCB/EECS, vol. 28, p. 2009, 2009.

[6] C. Liu, R. Ranjan, C. Yang, X. Zhang, L. Wang, and J. Chen, "MuR-DPA: Top-down levelled multireplica merkle hash tree based secure public auditing for dynamic big data storage on cloud," IEEE Transactions on Computers, vol. 64, pp. 2609-2622, 2014.

[7] D. D. Luxton, R. A. Kayl, and M. C. Mishkind, "mHealth data security: The need for HIPAA-compliant 
standardization," Telemedicine and e-Health, vol. 18, pp. 284-288, 2012.

[8] L. Jiang, Y. Cao, C. Yuan, X. Sun, and X. Zhu, "An effective comparison protocol over encrypted data in cloud computing," Journal of Information Security and Applications, vol. 48, p. 102367, 2019.

[9] S. Singh and I. Chana, "QoS-aware autonomic resource management in cloud computing: a systematic review," ACM Computing Surveys (CSUR), vol. 48, p. 42, 2016.

[10] J. Yu and R. Buyya, "A taxonomy of workflow management systems for grid computing," Journal of Grid Computing, vol. 3, pp. 171-200, 2005.

[11] F. Zafar, A. Khan, S. U. R. Malik, M. Ahmed, A. Anjum, M. I. Khan, et al., "A survey of cloud computing data integrity schemes: Design challenges, taxonomy and future trends," Computers \& Security, vol. 65, pp. 29-49, 2017.

[12] K. Compton and S. Hauck, "Reconfigurable computing: a survey of systems and software," $A C M$ Computing Surveys (csuR), vol. 34, pp. 171-210, 2002.

[13] B. Di Martino, M. Rak, M. Ficco, A. Esposito, S. Maisto, and S. Nacchia, "Internet of things reference architectures, security and interoperability: A survey," Internet of Things, vol. 1, pp. 99-112, 2018.

[14] S. S. Gill, P. Garraghan, V. Stankovski, G. Casale, R. K. Thulasiram, S. K. Ghosh, et al., "Holistic resource management for sustainable and reliable cloud computing: An innovative solution to global challenge," Journal of Systems and Software, 2019.

[15] A. Nadjaran Toosi, Richard O. Sinnott, and R. Buyya, "Resource provisioning for data-intensive applications with deadline constraints on hybrid clouds using Aneka," Future Generation Computer Systems, vol. 79, pp. 765-775, 2018/02/01/2018.

[16] N. Jindal, S. Chandran, P. R. Panda, S. Prasad, A. Mitra, K. Singhal, et al., "DHOOM: Reusing Designfor-Debug Hardware for Online Monitoring," in Proceedings of the 56th Annual Design Automation Conference 2019, 2019, p. 99.

[17] H. Suleman, "Utility-based High Performance Digital Library Systems," in Proceedings of the SecondWorkshop on Very Large Digital Libraries VLDL2009 A Workshop in conjunction with the European Conference on Digital Libraries 2009, 2009.

[18] M. Singh, "A Comparative Study on Grid Computing and Cloud Computing," no. June, pp. 708-713, 2015.

[19] R. Friedman, K. P. Birman, S. Keshav, and W. Vogels, "Reliable time delay-constrained cluster computing," ed: Google Patents, 2002.

[20] E. Yiannis and G. Joseph, "Introduction to Grid'5000," 2011.

[21] Y. Li and Z. Lan, "Exploit failure prediction for adaptive fault-tolerance in cluster computing," in Sixth IEEE International Symposium on Cluster Computing and the Grid (CCGRID'06), 2006, pp. 8 pp.-538.

[22] M. Li, D. Goldberg, W. Tao, and Y. Tamir, "Fault-tolerant cluster management for reliable highperformance computing," in Int. Conf. on Parallel and Distributed Computing and Systems, 2001, pp. 480-485.

[23] A. Jain and R. Singh, "An innovative approach of Ant Colony optimization for load balancing in peer to peer grid environment," in 2014 International Conference on Issues and Challenges in Intelligent Computing Techniques (ICICT), 2014, pp. 1-5.

[24] D. Kahanwal and D. T. Singh, "The distributed computing paradigms: P2P, grid, cluster, cloud, and jungle," arXiv preprint arXiv:1311.3070, 2013.

[25] J. Clabby and C. Analytics, "The Grid report, 2004 edition," Technical report, Clabby Analytics2004.

[26] F. Dong and S. G. Akl, "Scheduling algorithms for grid computing: State of the art and open problems," Technical report2006.

[27] D. S. Milojicic, V. Kalogeraki, R. Lukose, K. Nagaraja, J. Pruyne, B. Richard, et al., "Peer-to-peer computing," ed: Technical Report HPL-2002-57, HP Labs, 2002.

[28] M. Arumugam, A. P. Sheth, and I. B. Arpinar, "Towards peer-to-peer semantic web: A distributed environment for sharing semantic knowledge on the web," 2002.

[29] L. Napster, "Napster," URL: http://www. napster. com, 2001.

[30] M. Almorsy, J. Grundy, and I. Müller, "An analysis of the cloud computing security problem," arXiv preprint arXiv:1609.01107, 2016.

[31] G. Feuerlicht and S. Govardhan, "Impact of cloud computing: beyond a technology trend," Systems integration, pp. 262-269, 2010.

[32] C. Weinhardt, A. Anandasivam, B. Blau, N. Borissov, T. Meinl, W. Michalk, et al., "Cloud computinga classification, business models, and research directions," Business \& Information Systems Engineering, vol. 1, pp. 391-399, 2009.

[33] A. Davis, J. Parikh, and W. E. Weihl, "Edgecomputing: extending enterprise applications to the edge of the internet," in Proceedings of the 13th international World Wide Web conference on Alternate track papers \& posters, 2004, pp. 180-187. 
[34] H. Pang and K.-L. Tan, "Authenticating query results in edge computing," in Proceedings. 20th International Conference on Data Engineering, 2004, pp. 560-571.

[35] C. Canali, M. Rabinovich, and Z. Xiao, "Utility computing for Internet applications," in Web Content Delivery, ed: Springer, 2005, pp. 131-151.

[36] G. Roussos and V. Kostakos, "RFID in pervasive computing: state-of-the-art and outlook," Pervasive and Mobile Computing, vol. 5, pp. 110-131, 2009.

[37] M. Weiser, "Some computer science issues in ubiquitous computing," Communications of the ACM, vol. 36, pp. 75-84, 1993.

[38] M. Weiser, "Ubiquitous computing," in ACM Conference on Computer Science, 1994.

[39] M. Weiser, "Some computer science issues in ubiquitous computing," ACM SIGMOBILE Mobile Computing and Communications Review, vol. 3, p. 12, 1999.

[40] M. Weiser, R. Gold, and J. S. Brown, "The origins of ubiquitous computing research at PARC in the late 1980s," IBM systems journal, vol. 38, pp. 693-696, 1999.

[41] D. Saha and A. Mukherjee, "Pervasive computing: a paradigm for the 21 st century," Computer, vol. 36, pp. 25-31, 2003.

[42] F. J. Seinstra, J. Maassen, R. V. Van Nieuwpoort, N. Drost, T. Van Kessel, B. Van Werkhoven, et al., "Jungle computing: Distributed supercomputing beyond clusters, grids, and clouds," in Grids, Clouds and Virtualization, ed: Springer, 2011, pp. 167-197.

[43] N. Drost, J. Maassen, M. A. van Meersbergen, H. E. Bal, F. I. Pelupessy, S. P. Zwart, et al., "Highperformance distributed multi-model/multi-kernel simulations: A case-study in jungle computing," in 2012 IEEE 26th International Parallel and Distributed Processing Symposium Workshops \& PhD Forum, 2012, pp. 150-162.

[44] S. A. Ahson and M. Ilyas, Cloud computing and software services: theory and techniques: CRC Press, 2010.

[45] Z. Wu, Service Computing: Concept, Method and Technology: Academic Press, 2014.

[46] R. Buyya, C. S. Yeo, and S. Venugopal, "Market-oriented cloud computing: Vision, hype, and reality for delivering it services as computing utilities," in 2008 10th IEEE International Conference on High Performance Computing and Communications, 2008, pp. 5-13.

[47] D. Marr, "Artificial intelligence_-a personal view," Artificial Intelligence, vol. 9, pp. 37-48, 1977.

[48] M. Cheng, J. Li, and S. Nazarian, "DRL-cloud: Deep reinforcement learning-based resource provisioning and task scheduling for cloud service providers," in Proceedings of the 23rd Asia and South Pacific Design Automation Conference, 2018, pp. 129-134.

[49] D. Talia, "Cloud Computing and Software Agents: Towards Cloud Intelligent Services," in WOA, 2011, pp. 2-6.

[50] S. Singh and I. Chana, "QRSF: QoS-aware resource scheduling framework in cloud computing," The Journal of Supercomputing, vol. 71, pp. 241-292, 2015.

[51] S. J. Russell and P. Norvig, Artificial intelligence: a modern approach: Malaysia; Pearson Education Limited, 2016.

[52] A. Dawoud, S. Shahristani, and C. Raun, "Deep learning and software-defined networks: towards secure Iot architecture," Internet of Things, vol. 3, pp. 82-89, 2018.

[53] A. A. Alli and M. M. Alam, "SecOFF-FCIoT: Machine learning based secure offloading in Fog-Cloud of things for smart city applications," Internet of Things, vol. 7, p. 100070, 2019.

[54] S. S. Gill and R. Buyya, "A taxonomy and future directions for sustainable cloud computing: 360 degree view," ACM Computing Surveys (CSUR), vol. 51, p. 104, 2018.

[55] D. Gil, A. Ferrández, H. Mora-Mora, and J. Peral, "Internet of things: A review of surveys based on context aware intelligent services," Sensors, vol. 16, p. 1069, 2016.

[56] J. Gubbi, R. Buyya, S. Marusic, and M. Palaniswami, "Internet of Things (IoT): A vision, architectural elements, and future directions," Future generation computer systems, vol. 29, pp. 1645-1660, 2013.

[57] E. M. Tordera, X. Masip-Bruin, J. Garcia-Alminana, A. Jukan, G.-J. Ren, J. Zhu, et al., "What is a fog node a tutorial on current concepts towards a common definition," arXiv preprint arXiv:1611.09193, 2016.

[58] J. Lin, W. Yu, N. Zhang, X. Yang, H. Zhang, and W. Zhao, "A survey on internet of things: Architecture, enabling technologies, security and privacy, and applications," IEEE Internet of Things Journal, vol. 4, pp. 1125-1142, 2017.

[59] F. Bonomi, R. Milito, J. Zhu, and S. Addepalli, "Fog computing and its role in the internet of things," in Proceedings of the first edition of the MCC workshop on Mobile cloud computing, 2012, pp. 13-16.

[60] S. Singh, I. Chana, and M. Singh, "The journey of QoS-aware autonomic cloud computing," IT Professional, vol. 19, pp. 42-49, 2017.

[61] S. N. Shirazi, A. Gouglidis, A. Farshad, and D. Hutchison, "The extended cloud: Review and analysis of 
mobile edge computing and fog from a security and resilience perspective," IEEE Journal on Selected Areas in Communications, vol. 35, pp. 2586-2595, 2017.

[62] S. S. Gill, R. C. Arya, G. S. Wander, and R. Buyya, "Fog-Based Smart Healthcare as a Big Data and Cloud Service for Heart Patients Using IoT," in International Conference on Intelligent Data Communication Technologies and Internet of Things, 2018, pp. 1376-1383.

[63] A. M. Rahmani, T. N. Gia, B. Negash, A. Anzanpour, I. Azimi, M. Jiang, et al., "Exploiting smart eHealth gateways at the edge of healthcare Internet-of-Things: A fog computing approach," Future Generation Computer Systems, vol. 78, pp. 641-658, 2018.

[64] X. Fang, S. Misra, G. Xue, and D. Yang, "Smart grid-The new and improved power grid: A survey," IEEE communications surveys \& tutorials, vol. 14, pp. 944-980, 2011.

[65] J. Lin, W. Yu, and X. Yang, "Towards multistep electricity prices in smart grid electricity markets," IEEE Transactions on Parallel and Distributed Systems, vol. 27, pp. 286-302, 2015.

[66] C.-M. Chung, C.-C. Chen, W.-P. Shih, T.-E. Lin, R.-J. Yeh, and I. Wang, "Automated machine learning for Internet of Things," in 2017 IEEE International Conference on Consumer Electronics-Taiwan (ICCETW), 2017, pp. 295-296.

[67] L. Atzori, A. Iera, and G. Morabito, "The internet of things: A survey," Computer networks, vol. 54, pp. 2787-2805, 2010.

[68] S. Tuli, R. Mahmud, S. Tuli, and R. Buyya, "Fogbus: A blockchain-based lightweight framework for edge and fog computing," Journal of Systems and Software, 2019.

[69] S. A. Osia, A. S. Shamsabadi, A. Taheri, K. Katevas, S. Sajadmanesh, H. R. Rabiee, et al., "A hybrid deep learning architecture for privacy-preserving mobile analytics," arXiv preprint arXiv:1703.02952, 2017.

[70] K. K. Nguyen, D. T. Hoang, D. Niyato, P. Wang, D. Nguyen, and E. Dutkiewicz, "Cyberattack detection in mobile cloud computing: A deep learning approach," in 2018 IEEE Wireless Communications and Networking Conference (WCNC), 2018, pp. 1-6.

[71] S. Teerapittayanon, B. McDanel, and H.-T. Kung, "Distributed deep neural networks over the cloud, the edge and end devices," in 2017 IEEE 37th International Conference on Distributed Computing Systems (ICDCS), 2017, pp. 328-339.

[72] A. E. Eshratifar and M. Pedram, "Energy and performance efficient computation offloading for deep neural networks in a mobile cloud computing environment," in Proceedings of the 2018 on Great Lakes Symposium on VLSI, 2018, pp. 111-116.

[73] P. Li, J. Li, Z. Huang, C.-Z. Gao, W.-B. Chen, and K. Chen, "Privacy-preserving outsourced classification in cloud computing," Cluster Computing, vol. 21, pp. 277-286, 2018.

[74] J. Son and R. Buyya, "A taxonomy of software-defined networking (sdn)-enabled cloud computing," ACM Computing Surveys (CSUR), vol. 51, p. 59, 2018.

[75] J. Son and R. Buyya, "Priority-aware VM allocation and network bandwidth provisioning in softwaredefined networking (SDN)-enabled clouds," IEEE Transactions on Sustainable Computing, vol. 4, pp. 17-28, 2018.

[76] A. N. Toosi and R. Buyya, "Acinonyx: Dynamic Flow Scheduling for Virtual Machine Migration in SDNenabled Clouds," in 2018 IEEE Intl Conf on Parallel \& Distributed Processing with Applications, Ubiquitous Computing \& Communications, Big Data \& Cloud Computing, Social Computing \& Networking, Sustainable Computing \& Communications (ISPA/IUCC/BDCloud/SocialCom/SustainCom), 2018, pp. 886-894.

[77] T. He, A. N. Toosi, and R. Buyya, "Performance evaluation of live virtual machine migration in SDNenabled cloud data centers," Journal of Parallel and Distributed Computing, vol. 131, pp. 55-68, 2019.

[78] A. N. Toosi, J. Son, and R. Buyya, "Clouds-pi: A low-cost raspberry-pi based micro data center for software-defined cloud computing," IEEE Cloud Computing, vol. 5, pp. 81-91, 2018.

[79] R. Guha and D. Al-Dabass, "Impact of web 2.0 and cloud computing platform on software engineering," in 2010 International Symposium on Electronic System Design, 2010, pp. 213-218.

[80] W. A. Simm, F. Samreen, R. Bassett, M. A. Ferrario, G. Blair, J. Whittle, et al., "Se in es: Opportunities for software engineering and cloud computing in environmental science," in Proceedings of the 40th International Conference on Software Engineering: Software Engineering in Society, 2018, pp. 61-70.

[81] I. Scirlet, "Cloud Technology in the era of IoT, Blockchain, Machine Learning and AI," 2018.

[82] L. S. Bishop, A. W. Cross, I. F. Sertage, and J. M. Gambetta, "Job processing in quantum computing enabled cloud environments," ed: Google Patents, 2019.

[83] M. Caleffi, A. S. Cacciapuoti, and G. Bianchi, "Quantum internet: from communication to distributed computing!," arXiv preprint arXiv:1805.04360, 2018.

[84] M. Schuld, I. Sinayskiy, and F. Petruccione, "An introduction to quantum machine learning," Contemporary Physics, vol. 56, pp. 172-185, 2015. 
[85] M. N. Leuenberger and D. Loss, "Quantum computing in molecular magnets," Nature, vol. 410, p. 789, 2001.

[86] R. I. Ansari, H. Pervaiz, S. A. Hassan, C. Chrysostomou, M. A. Imran, S. Mumtaz, et al., "A New Dimension to Spectrum Management in IoT Empowered 5G Networks," IEEE Network, 2019.

[87] D. S. Linthicum, Cloud computing and SOA convergence in your enterprise: a step-by-step guide: Pearson Education, 2009.

[88] S. S. Gill, S. Tuli, M. Xu, I. Singh, K. V. Singh, D. Lindsay, et al., "Transformative Effects of IoT, Blockchain and Artificial Intelligence on Cloud Computing: Evolution, Vision, Trends and Open Challenges," Internet of Things, p. 100118, 2019.

[89] J. G. Andrews, S. Buzzi, W. Choi, S. V. Hanly, A. Lozano, A. C. Soong, et al., "What will 5G be?," IEEE Journal on selected areas in communications, vol. 32, pp. 1065-1082, 2014.

[90] Z. M. Temesvári, D. Maros, and P. Kádár, "Review of Mobile Communication and the 5G in Manufacturing," Procedia Manufacturing, vol. 32, pp. 600-612, 2019/01/01/2019.

[91] S. S. Gill, P. Garraghan, and R. Buyya, "ROUTER: Fog enabled cloud based intelligent resource management approach for smart home IoT devices," Journal of Systems and Software, 2019.

[92] S. Singh and I. Chana, "A survey on resource scheduling in cloud computing: Issues and challenges," Journal of grid computing, vol. 14, pp. 217-264, 2016.

[93] S. Singh, I. Chana, and R. Buyya, "STAR: SLA-aware autonomic management of cloud resources," IEEE Transactions on Cloud Computing, 2017.

[94] S. Singh and I. Chana, "Consistency verification and quality assurance (CVQA) traceability framework for SaaS," in 2013 3rd IEEE International Advance Computing Conference (IACC), 2013, pp. 1-6.

[95] S. Singh and I. Chana, "Enabling reusability in agile software development," arXiv preprint arXiv:1210.2506, 2012.

[96] S. S. Gill and R. Buyya, "Bio-Inspired Algorithms for Big Data Analytics: A Survey, Taxonomy, and Open Challenges," in Big Data Analytics for Intelligent Healthcare Management, ed: Elsevier, 2019, pp. $1-17$.

[97] S. S. Gill, I. Chana, and R. Buyya, "IoT based agriculture as a cloud and big data service: the beginning of digital India," Journal of Organizational and End User Computing (JOEUC), vol. 29, pp. 1-23, 2017.

[98] A. R. Rao and D. Clarke, "Perspectives on emerging directions in using IoT devices in blockchain applications," Internet of Things, p. 100079, 2019.

[99] R. Buyya, S. N. Srirama, G. Casale, R. Calheiros, Y. Simmhan, B. Varghese, et al., "A manifesto for future generation cloud computing: research directions for the next decade," ACM computing surveys (CSUR), vol. 51, p. 105, 2018.

[100] A. Kaur, V. Singh, and S. S. Gill, "The future of cloud computing: opportunities, challenges and research trends," in 2018 2nd International Conference on I-SMAC (IoT in Social, Mobile, Analytics and Cloud)(ISMAC) I-SMAC (IoT in Social, Mobile, Analytics and Cloud)(I-SMAC), 2018 2nd International Conference on, 2018, pp. 213-219.

[101] A. Beloglazov and R. Buyya, "Optimal online deterministic algorithms and adaptive heuristics for energy and performance efficient dynamic consolidation of virtual machines in cloud data centers," Concurrency and Computation: Practice and Experience, vol. 24, pp. 1397-1420, 2012.

[102] S. S. Gill, R. Buyya, I. Chana, M. Singh, and A. Abraham, "BULLET: particle swarm optimization based scheduling technique for provisioned cloud resources," Journal of Network and Systems Management, vol. 26, pp. 361-400, 2018.

[103] M. Mukherjee, R. Matam, L. Shu, L. Maglaras, M. A. Ferrag, N. Choudhury, et al., "Security and privacy in fog computing: Challenges," IEEE Access, vol. 5, pp. 19293-19304, 2017. 